\title{
Interventional Treatment of a Patient with Acute Coronary Syndrome and Multivessel Coronary Artery Disease Who Refuses Cardiac Bypass Surgery
}

Juergen Kammler*, Hermann Blessberger, Klaus Kerschner, Alexander Kypta, Thomas Lambert and Clemens Steinwender

\author{
Medical Department- Cardiology, Linz General Hospital, Johannes Kepler University School of Medicine, Linz, Austria
}

Received: January 01, 2014; Accepted: June 23, 2014, Published: June 25, 2014

*Corresponding author: Juergen Kammler, Medical Department- Cardiology, Linz General Hospital, Johannes Kepler University School of Medicine, Linz, Austria, Tel: +43 7327806 73213; Fax: +43 7327806 6205; E-mail: juergen.kammler@akh.linz.at

\begin{abstract}
We report a case of an 82 year old female patient suffering from an acute coronary syndrome with angina pectoris at rest accompanied by electrocardiographic signs of ischemia.

After the patient was stabilized, she consecutively underwent coronary angiography. The examination revealed a three vessel disease involving the left main stem. Surgical revascularization by coronary artery bypass grafting was recommended. As the patient explicitly refused an operation but accepted a high risk left main stem trifurcation intervention, aggravated by an occluded right coronary artery, we performed this intervention successfully during a second procedure.
\end{abstract}

This case shows, that high risk interventions are feasible if perfomed by a skilled cardiologist and an experienced staff at a high volume interventional catheterization center.

Keywords: Acute coronary syndrome; Left main stem; Coronary intervention; Trifurcation lesion.

\section{Abbreviations}

ECG: Electrocardiogram; LAD: Left Anterior Descending Coronary Artery; RI: Ramus Intermedius; LCX: Left Circumflex Artery; LM: Left main stem

\section{Introduction}

Revascularization in patients suffering from acute coronary syndromes (ACS) leads to a reduction in mortality. This holds true for percutaneous coronary intervention (PCI) as well as for coronary artery bypass grafting (CABG) $[1,2]$.CABG is still regarded as the standard of care for significant left main (LM) disease in patients eligible for surgery (indication level I, level of evidence A), whereas the 2008 ESC guidelines on PCI state that stenting for unprotected LM disease should only be considered in the absence of other revascularization options (II B) $[3,4]$. However, in daily clinical practice patients are often reluctant to undergo the recommended CABG and ask for alternative therapy strategies like PCI. We report a challenging interventional case performed in a patient with ACS and coronary multivessel disease involving the LM who unambiguously refused CABG and subsequently underwent complex PCI.

\section{Case Report}

We report the case of an 82 year old female patient suffering from typical angina pectoris CCS class II since 6 months.

At the day of admission she reported severe thoracic discomfort lasting for 6 hours. The patient's ECG (Figure 1) at admission showed ischemic ST-depression in the anteroseptal leads with a slight ST-segment elevation in the lead AVR. The patient was on $100 \mathrm{mg}$ aspirin once daily, a statin and a beta-blocker (metoprolol succinate). Upon admission, a $600 \mathrm{mg}$ clopidogrel loading dose and benzodiazepines were given orally, and morphine hydrochloride and nitrates were administered parenterally resulting in clinical stabilization within 30 minutes. The patient's physical examination was unremarkable. The patient's history revealed coronary artery disease with bare-metal stent implantation of the proximal Left Anterior Descending Coronary Artery (LAD) eight years ago and hypercholesterolemia on treatment as relevant cardiovascular risk factors. Furthermore, strumectomy of a retrosternal struma nodosa with sternotomy had been performed twelve years ago.

Transthoracic echocardiography showed a moderately reduced left ventricular systolic function (ejection fraction $48 \%$ as calculated by the biplane Simpson's method) with an akinesia of the inferior wall.

Lab examinations showed elevated troponin $\mathrm{T}$ values (0.335 ng/ml-upper limit of normal: $0.014 \mathrm{ng} / \mathrm{ml}$ ) and hypercholesterolemia $(230 \mathrm{mg} / \mathrm{dl}-$ upper limit of normal: 200 $\mathrm{mg} / \mathrm{dl}), \mathrm{LDL}-\mathrm{hypercholesterolemia}(143 \mathrm{mg} / \mathrm{dl}-$ desired values in coronary artery disease below $70 \mathrm{mg} / \mathrm{dl}$ ). Red blood cell and platelet counts as well as the renal function were normal. The patient underwent coronary angiography which revealed a severe multivessel disease with a chronic occluded Right Coronary Artery (RCA) and a subtotal stenosis of the distal Left 


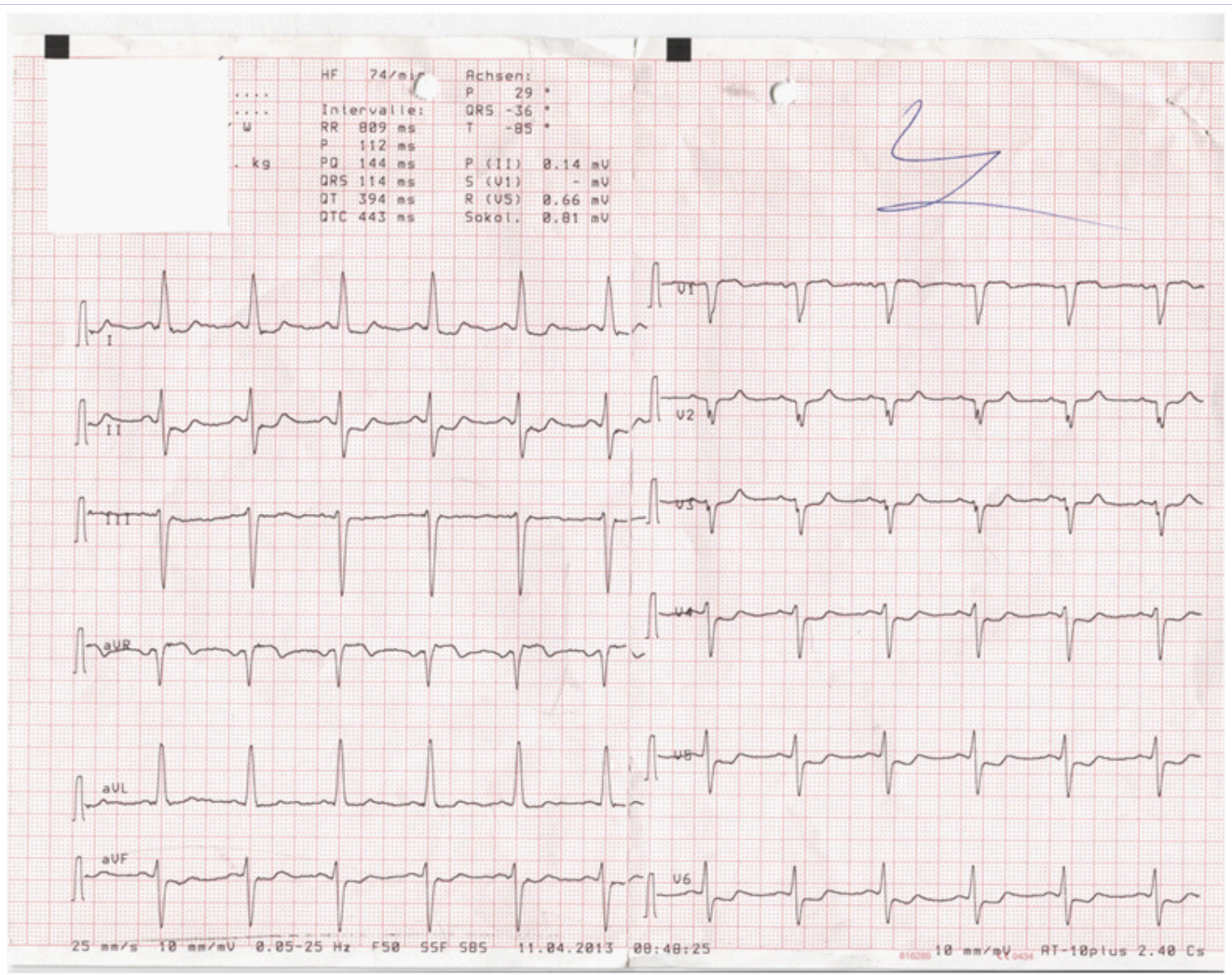

\section{ECG at admission}

Figure 1: Sinus rhythm, 75 beats per minute, anteroseptal ischemic ST-segment depression, discrete ST-segment elevation in aVR.

Main (LM) involving the origin of the LAD, left circumflex (LCX) and a large ramus intermedius (RI) in terms of a trifurcational lesion [Moving image 1].

No in-stent restinosis in the proximal LAD but a non-significant luminal narrowing in the mid section of the vessel was found. The LCX showed an additional significant stenosis in its mid-section (Figure 2). We recommended surgical revascularization by CABG, as indicated in current guidelines $[3,4]$.

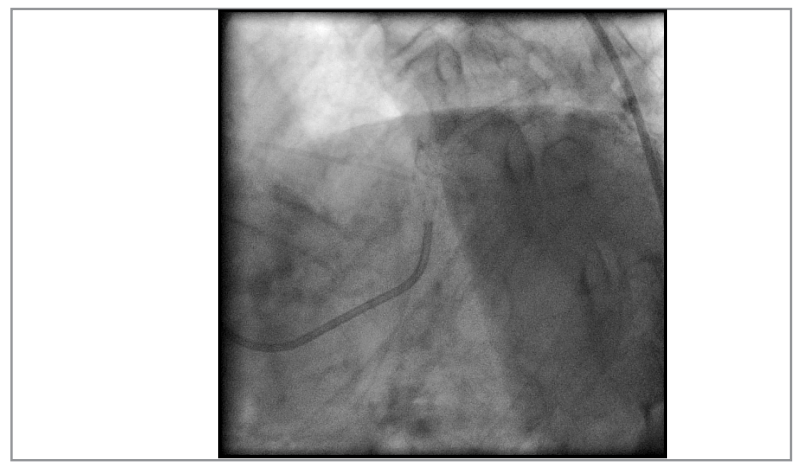

Moving image 1: Diagnostic angiogram in left anterior oblique projection showing severe three vessel disease involving the LM.

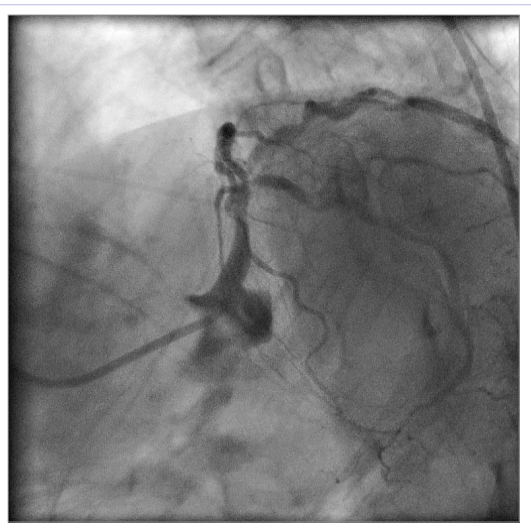

Figure 2: Diagnostic angiogram of the left coronary artery showed a severe multivessel disease with a subtotal distal LM stem stenosis involving the origin of the LAD, LCX and a large RI in terms of a trifurcationallesion as well as a chronically occluded right coronary artery.

As the patient explicitly refused an operation but accepted a high risk intervention, we scheduled her for a complex coronary intervention the next day. As the small RCA was chronically occluded, we decided not to try to re-open the vessel, but planned to intervene the distal LM trifurcation stenosis. Using a 
right femoral access, a 7 French 4.0 guiding catheter (Launcher ${ }^{\circledR}$ Coronary Guide Catheter; Medtronic, MN, USA) was positioned in the ostium of the left coronary artery. 5.000 IU of heparin were administered intravenously before the intervention started.

Then three Run through ${ }^{\circledR}$ guide wires (Terumo; Leuven, Belgium) were positioned in the LAD, LCX and RI.

\section{First step: Ostial stenosis of the LAD/ RI}

Sequential balloon dilatations of the distal LM into the LAD and the origin of the RI were performed, respectively, followed by stent implantations of the LAD and distal LM $(3.0 \times 22 \mathrm{~mm})$ and RI $(2.75 \times 12 \mathrm{~mm})$ in mini-crush technique (all stents used: Resolute Integrity ${ }^{\circledR}$ zotarolimus eluting stents; Medtronic; MN, USA, with implantation pressures up to 16 atmospheres) [5,6]. As a TIMI III flow was assessed in both vessels after stent implantation, no final kissing-balloon dilatation was performed.

\section{Second step: Ostial stenosis and stenosis of the mid- section of the LCX}

[Moving image 2]: A kissing-balloon dilatation of the LM/LAD and LCX was performed. As the result was not ideal, two stents were implanted. One in the mid-section $(2.25 \times 12 \mathrm{~mm})$ of the LCX and another one at the origin of LCX $(2.25 \times 8 \mathrm{~mm})$ in T-stenting technique [7] with the implanted LM/LAD stent (Figure 3). A final kissing-balloon dilatation led to a discrete dissection of the midsection of the LM.

\section{Third step: Treating the LM dissection}

[Moving image 3]: To seal the dissection, a $3.5 \times 15 \mathrm{~mm}$ stent was implanted into the LM, slightly overlapping with the stent in the distal LM/LAD.

\section{Fourth step: Peri-interventional emergency}

However, this stent implantation resulted in an occlusion of the RI (Figure 4) with signs of ischemia on the ECG and symptoms of angina. Because of suspected thrombi at the stented parts of the LAD and the RI, a weight-adapted bolus of abciximab was administered intravenously $(0.25 \mathrm{mg} / \mathrm{kg}=8.6 \mathrm{ml})$.

Afterwards, the RI was rewired (Pilot 50®; Abbott Vascular, ILL, USA). A balloon dilatation of the ostium of the RI using a $2.75 \mathrm{~mm}$ non-compliant balloon (Beo $\AA$; SIS Medical, Switzerland),

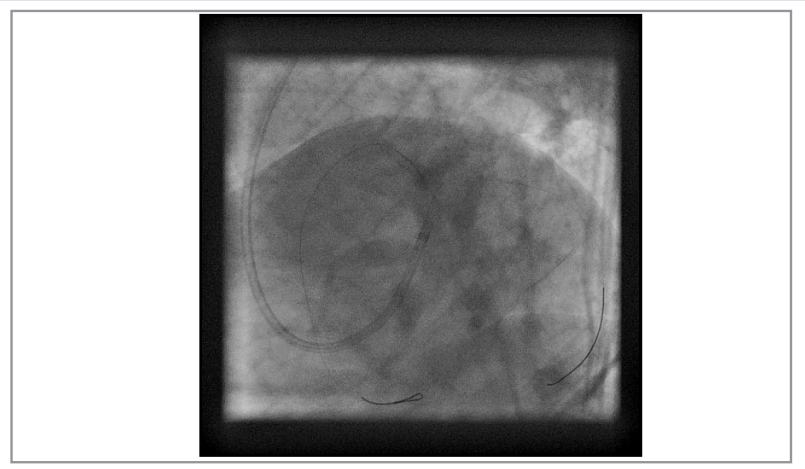

Moving image 2: Result after stenting the mid-section of the LCX. Occurrence of a spasm distal of the implanted stent.

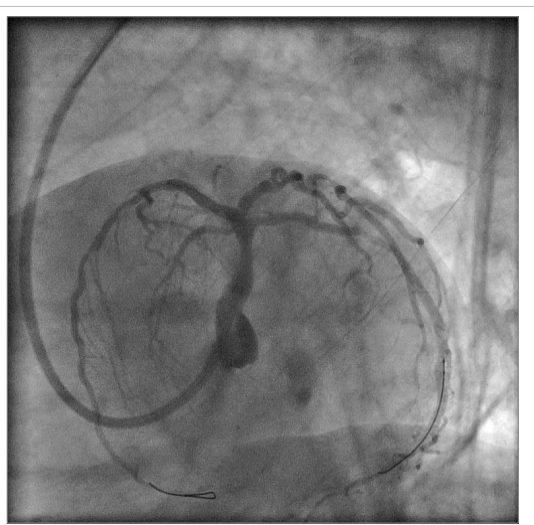

Figure 3: Result after „mini- crush” stenting of the LAD/RI as well as T-stenting of the LAD/LCX.

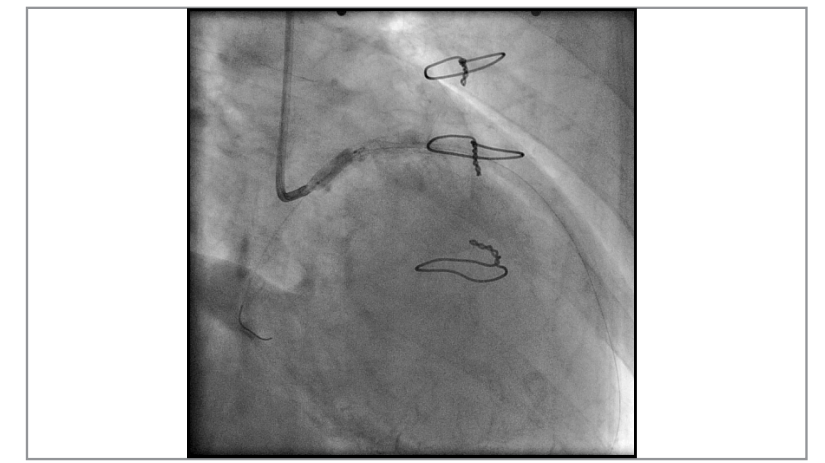

Moving image 3: Stenting of the LM.

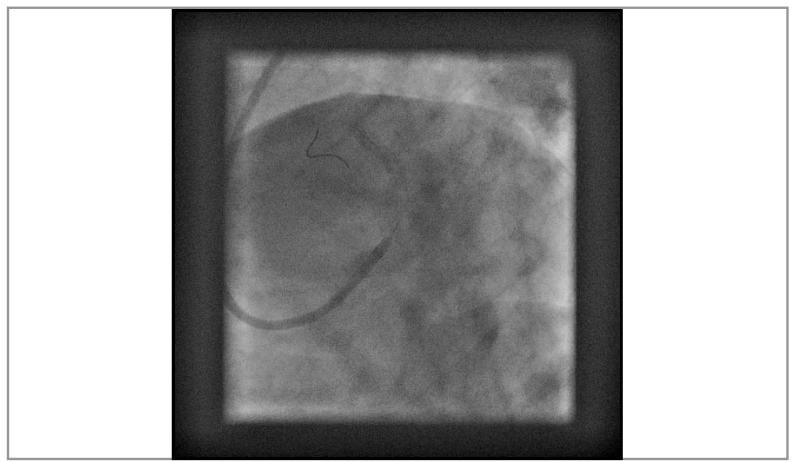

Moving image 4: Emergency: RI occlusion.

followed by a kissing-balloon dilatation of the LAD and RI (3.0 $\mathrm{mm} / 2.75 \mathrm{~mm}$ balloon), led to a restoration of TIMI III flow in all three vessels (Moving image 5), accompanied by normalization of the ECG (Figure 5) and disappearance of clinical symptoms.

After the intervention, the patient did well and could be discharged on the second day after the procedure.

She is now on dual antithrombotic medication comprising acetylsalicylic acid (100 mg/day) and clopidogrel (75mg/day), a beta-blocker (metaprolol succinate $95 \mathrm{mg} /$ day) and an AT I-antagonist (candesartan hydrochlorothiazide 16/12.5 mg/day) and fluvastatin $40 \mathrm{mg} /$ day. 


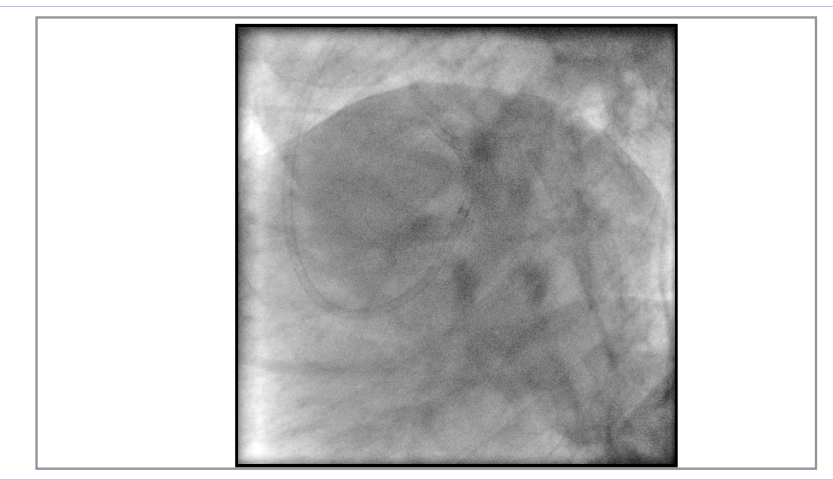

Moving image 5: Final result after complex intervention.

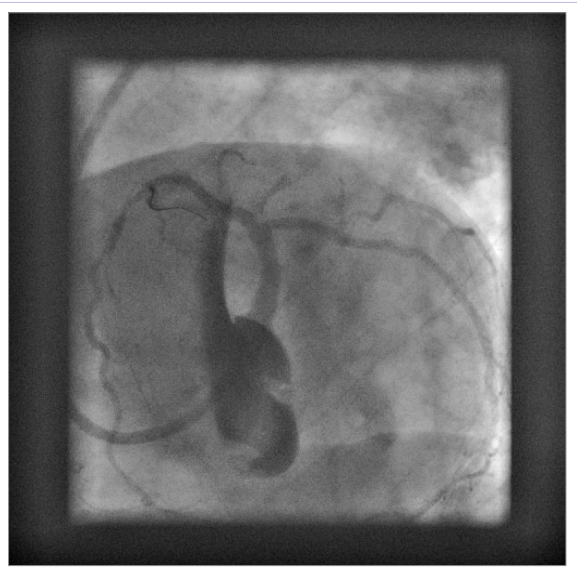

Figure 4: Emergency after stenting the LM dissection- occlusion of the RI.

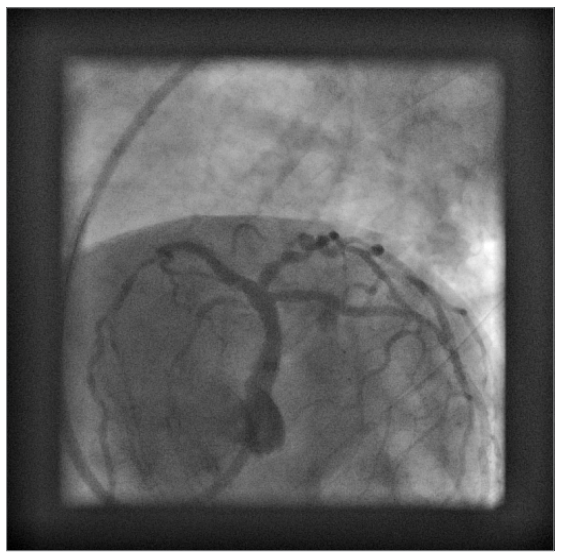

Figure 5: Result after complex intervention.

A clinical check-up at our outpatients' clinic three months after intervention as well as an angiographic control after further three months are scheduled.

\section{Discussion}

According to current guidelines, patients with significant multivessel coronary artery disease involving the LM should undergo CABG $[3,4]$. This recommendation is partially derived from the landmark trial SYNTAX which showed that patients had lower event rates after CABG than patients treated with PCI. The higher event rate in the PCI group was mainly caused by the higher rate of re-interventions compared to the CABG group during follow-up. Patients with CABG had higher rates of cerebrovascular events, especially in the first year after surgery [8]. However, two important points have to be made here: First, the SYNTAX trial used a first-generation drug-eluting stent (DES; TAXUS $^{\circledR}$; Boston Scientific, MN, USA) with a known higher rate of in-stent restenosis compared to newer stent generations which might have led to much better results in patients treated with PCI $[9,10]$. Second, events like re-interventions and cerebrovascular events were subsumed as a combined endpoint which made no further quantitative or qualitatative differentiation. In our opinion, these differences are relevant for the patients treated (who might accept an interventional re-intervention but want to avoid a cerebrovascular event). Guidelines and recommendations are important for clinical physicians in everyday practice. Nevertheless, the patients' decisions have to be respected too. If patients want to be treated in a way different to guideline recommendations, we have to accept their choice. In our specific case, the patient refused a surgical intervention despite being a class I indication and preferred PCI instead which is only classified as II B. As the armamentarium in the cath labs today is continuously getting better as stent designs and catheter materials are steadily improved and there is more routine in performing complex PCIs nowadays, we think that a decision like ours in this very case is reasonable and proved to be safe. Nevertheless, we have to stress the fact that skilled interventional cardiologists and experienced staff are essential to perform such high risk interventions.

\section{Conclusion}

In conclusion, this case highlights 3 important points:

1. High risk interventions are feasible if they are performed by skilled cardiologists and an experienced staff at a high volume interventional catheterization center.

2. Even in cases where a surgical approach is clearly recommended, percutaneous intervention is a possible and an effective solution for patients refusing for an operation. However, an appropriate patient education regarding the potential risks and the importance of adherence to the post-interventional medical therapy is crucial.

3. Intravascular imaging was not available at the time of intervention due to technical reasons. However, this case shows that even complex coronary interventions can be performed without additional imaging modalities such as optical coherence tomography or intravascular ultrasound by a skilled interventional cardiologist. Good angiographical results can be achieved by high pressure stent implantation and subsequent kissing-balloon dilatation.

\section{References}

1. Andersen HR, Nielsen TT, Rasmussen K, Thuesen L, Kelbaek H, Thayssen $\mathrm{P}$, et al. DANAMI-2 Investigators:A comparison of coronary angioplasty with fibrinolytic therapy in acute myocardial infarction. $\mathrm{N}$ 
Engl J Med. 2003; 349(8): 733-742.

2. Cannon CP, Weintraub WS, Demopoulos LA, Vicari R, Frey MJ, Lakkis $\mathrm{N}$, et al. TACTICS (Treat Angina with Aggrastat and Determine Cost of Therapy with an Invasive or Conservative Strategy)-Thrombolysis in Myocardial Infarction 18 Investigators. Comparison of early invasive and conservative strategies in patients with unstable coronary syndromes treated with the glycoprotein IIb/IIIa inhibitor tirofiban. N Engl J Med. 2001; 344(25): 1879-1887.

3. Wijns W, Kolh P, Danchin N, Di Mario C, Falk V, Folligue T, et al. The Task Force on Myocardial Revascularization of the European Society of Cardiology (ESC) and the Association for Percutaneous Cardiovascular Interventions (EAPCI). Guidelines on myocardial revascularization. European Heart Journal. 2010; 31(20): 2501-55. doi: 10.1093/eurheartj/ehq277.

4. Hillis LD, Smith PK, Anderson JL, Bittl JA, Bridges CR, Byrne JG, et al. American College of Cardiology Foundation; American Heart Association Task Force on Practice Guidelines; American Association for Thoracic Surgery; Society of Cardiovascular Anesthesiologists; Society of Thoracic Surgeons; 2011 ACCF/AHA Guideline for Coronary Artery Bypass GraftSurgery: A report of the American College of Cardiology Foundation/American Heart Association Task Force on Practice Guidelines: Developed in collaboration with the American Association for Thoracic Surgery, Society of Cardiovascular Anesthesiologists, and Society of Thoracic Surgeons. J Am Coll Cardiol. 2011; 58(24): e123-210. doi: 10.1016/j.jacc.2011.08.009.

5. Galassi AR, Colombo A, Buchbinder M, Grasso C, Tomasello SD, Ussia GP, Tamburino C, etal. Long-term outcomes of bifurcation lesions after implantation of drug-eluting stents with the "mini-crush technique". Catheter Cardiovasc Interv. 2007; 69(7): 976-983.
6. Sheiban I, Albiero R, Marsico F, Dharmadhikari A, Tzifos V, Pagnotta P, et al. Immediate and long-term results of " $T^{\prime \prime}$ stenting for bifurcation coronary lesions. Am J Cardiol. 2000May1; 85(9): 1141-1144.

7. Colombo A, Stankovic G, Orlic D, Corvaja N, Liistro F, Airoldi F, et al. Modified T-stenting technique with crushing for bifurcation lesions: immediate results and 30-day outcome. Catheter Cardiovasc Interv. 2003; 60(2): 145-151.

8. Mohr FW, Morice MC, Kappetein AP, Feldman TE, Stahle E, Colombo A, et al. Coronary artery bypass graft surgery versus percutaneous coronary intervention in patients with three-vessel disease and left main coronary disease: 5-year follow-up of the randomised, clinical SYNTAX trial. Lancet. 2013; 381(9867): 629-638. doi: 10.1016/ S0140-6736(13)60141-5.

9. Stone GW, Ellis SG, Cox DA, Hermiller J, O'Shaughnessy C, Mann JT, et al. TAXUS-IV Investigators:One-year clinical results with the slowrelease, polymer-based, paclitaxel-eluting TAXUS stent: the TAXUS-IV trial. Circulation. 2004; 109(16): 1942-1947.

10. Kereiakes DJ, Sudhir K, Hermiller JB, Gordon PC, Ferguson J, Yaqub $\mathrm{M}$, et al. Comparison of everolimus-eluting and paclitaxel-eluting coronary stents in patients undergoing multilesion and multivessel intervention: the SPIRIT III (A Clinical Evaluation of the Investigational Device XIENCE V Everolimus Eluting Coronary Stent System [EECSS] in the Treatment of Subjects With De Novo Native Coronary Artery Lesions) and SPIRIT IV (Clinical Evaluation of the XIENCE V Everolimus Eluting Coronary Stent System in the Treatment of Subjects With De Novo Native Coronary Artery Lesions) randomized trials. JACC Cardiovasc Interv. 2010; 3(12): 1229-39. doi: 10.1016/j. jcin.2010.09.014. 